\title{
ALGUNS ASPECTOS SEMÂNTICO/ SINTÁTICOS DA TRANSITIVIDADE EM \\ IBÍBIO
}

Márcia Santos Duarte de Oliveira *

\section{Introdução}

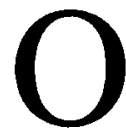

povo ibíbio, falante da língua denominada ibíbio, é originário da Nigéria, oeste da África. A língua ibíbio é classificada como língua "LowerCross", da subfamília "Cross River", sub-ramo "East Benue-Congo", pertencente ao ramo "Proto Benue-Congo", do grande tronco lingüístico "NigerCongo", que abrange mais de mil línguas africanas. ${ }^{1}$

Urua (2000) insere a língua ibíbio entre 19 variedades de línguas "Lower Cross", traçadas a partir de uma fonte em comum chamada de Ibibiod por Essien (1990):

\section{Ibibiod $^{2}$}

Iko Ibeno Ibuoro Itu Mbonuso Ito Nkari Anaang Efik Ibibio

* Universidade Federal de São Paulo.

1 Williamson e Blench (2000).

2 Urua (2000, p. 3). 
Ukwa Ekit Oro Okobo Ebughu Ilue Ewang-Uda Ekit Etebi Mbo (Efai)

A fonte lbibiod visa a capturar o relacionamento genético dentro do grupo. Devido ao alto grau de mútua inteligibilidade entre os falantes dessas 19 línguas, a comunicação dentro do estado de Akwa Ibom e no estado vizinho, de Cross River, onde essas línguas são faladas (cf. anexo), não é muito problemática.

A língua ibíbio possui vários dialetos com diversos graus de inteligibilidade e ligados a diversos clãs, como se verifica a seguir:

\section{lbíbio $^{3}$}

central eket oron ibeno okobo enyong itu mbon uso nkari

Os dados selecionados para este trabalho são baseados no dialeto ibíbio central.

Neste estudo, que investiga aspectos interativos entre a sintaxe e a semântica em ibíbio, a análise dos dados é baseada nos seguintes modelos teóricos: (i) sintático - "Programa minimalista" (Chomsky, 1995; 1998; 1999); (ii) semântico - "Semântica gerativa" (Heim; Kratzer, 1998).

O trabalho objetiva mostrar que os traços- $\varphi$ de concordância do sujeito -SAfs -, quando checados na projeção de Tempo (daqui em diante, TP), sempre permanecem visíveis em Forma Fonológica (daqui em diante, PF) como parte da morfologia verbal na língua ibíbio. Porém, os traços- $\varphi$ de concordância do objeto - OAfs - só permanecem visíveis em PF quando o complemento do Sintagma Determinante (daqui em diante, DP) possui o traço semântico [+HUMANO].

\section{Tipos de complementos em ibibio}

Essien (1990) observou que "...cada verbo em ibíbio é capaz de ter um objeto, ao menos um objeto do tipo cognato, ou seja, um objeto que é morfologicamente relacionado ou derivado do próprio verbo." 4

Sintagmas verbais em ibíbio (daqui em diante, VP) possuem dois tipos de complementos, como se pode ver na seção a seguir.

3 Oliveira (1995).

4 Essien (1990, p. 142, traduzido). 


\title{
Complementos derivados
}

Os complementos VP em ibíbio (chamados de "bound-complements" por alguns africanistas) $)^{5}$ são relacionados ou derivados do verbo como se vê em (1):

(1) (Enoo) ádọkkoó ńdọkkơo

(Eno) 3sSAf-falar fala $^{7}$

"Eno fala a fala / Eno é falante"

\section{Complementos não relacionados com o verbo (não derivados)}

Alguns complementos não são relacionados com o verbo ou derivados da forma verbal, como se verifica nos exemplos de complementos em (2) n $\Uparrow \mathbf{s o}$ 个o $\Leftrightarrow$

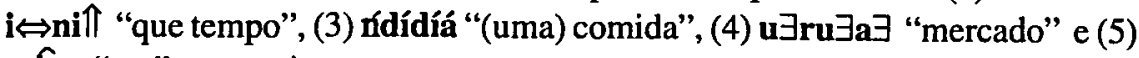
mien "me", respectivamente:

\author{
(Àfò) a- $\prod_{b i} \Downarrow_{\text {di }} \downarrow$ n $\Uparrow$ so $\|_{o} \Leftrightarrow \mathrm{i} \Leftrightarrow$ ni $\Uparrow$ ? \\ (2s) 2sSAf-futII-vir que tempo \\ "Você virá quando/ que tempo?" \\ (Èmè) á-nâm ñ́dídiá \\ (Eme) 3sSAf-cozinhar comida \\ "Eme está cozinhando (uma) comida"
}

5 Dickson (1995).

6 A ortografía da língua ibíbio usada neste estudo está de acordo com Essien (1983): a, b, d, e, $\mathfrak{\wp}, \mathbf{f}, \leftrightarrow$, gh, h, i, i, k, kp, m, n, n, nw, ny, o, o, p, r, s, t, u, u, w, y. A ortografia representa os seguintes fonemas: a representa $/ \mathbf{a} / ; \mathbf{b}$ representa $/ \mathbf{b} /$; d representa $/ \mathbf{d} /$; e representa $/ e / ; \wp$ representa $/ \wp / ; f$ representa $/ f / ; \boldsymbol{\partial}$ representa $/ \partial /$; $\boldsymbol{g}$ representa $/ \boldsymbol{y} /$ (um alofone de $h / x /) ; h$ representa $/ x / ; i$ representa $/ i / ; i$ representa $/ i / ; k$ representa $/ k / ; k p$ representa $/ k p / ; m$ representa $/ m / ; n$ representa $/ n / ; \tilde{n}$ representa $/ \boldsymbol{g} / ; n \boldsymbol{w}$ representa $/ \boldsymbol{g}^{\boldsymbol{w}} / ; \boldsymbol{n} \boldsymbol{y}$ representa $/ \boldsymbol{n} / ; \boldsymbol{o}$ representa $/ o / ; \boldsymbol{o}$ representa $/ o / ; p$ representa $/ p / ; r$ representa $/ r / ; s$ representa $/ s / ; t$ representa $/ t / ; u$ representa $/ u / ; u$ representa $/ \boldsymbol{u} / ; w$ representa $/ w / ; y$ representa $/ y /$.

Ibibio possui três tons de nível: /a $\Leftrightarrow /$ alto, /ađ/ baixo, / $\mathbf{a} \Leftrightarrow /$ 'downstep(ped)', e dois tons de contorno: /a $\downarrow /$ alto-baixo; /a $0 /$ baixo-alto (cf. Urua, 2000).

7 Abreviaturas: [DP] - Sintagma Determinante; [1s] - pronome de $1^{4}$ pessoa do singular; [2s] - pronome de $2^{a}$ pessoa do singular; [3s] - pronome de $3^{\mathrm{a}}$ pessoa do singular; [fut] - Future; [PF] - Forma Fonologica; [pst] - Passado; [T] - Tempo; [OAf] - traços- $\varphi$ de concordância do objeto; [SAf] - traços- $\varphi$ de concordância do sujeito. 
(Àfò) à-ka\& $\mathrm{u} \exists r u \exists a \exists$

(2s) 2sSAf-pstII-ir mercado

"Você vai ao mercado"

(Òkón) à-ké ìn-kóód (miên)

(Okon) 3sSAf-pstll lsOAf-chamar (1s)

"(Okon) chamou (me)"

Ndimele e Oliveira (2001) advogam que ibíbio é uma língua de sujeito nulo e que, além da omissão do sujeito de uma oração, também o objeto pode ser omitido, como visto no exemplo (5) acima.

Os exemplos (1)-(5) demonstram que os SAfs quando checados em TP sempre permanecem visíveis em $\mathrm{PF}^{8}$ Os OAfs, porém, permanecem visíveis em PF somente em dados como (5). Este aspecto da transitividade em ibíbio será demonstrado a seguir.

\section{Traços- $\varphi$ de concordância do objeto visíveis em pf}

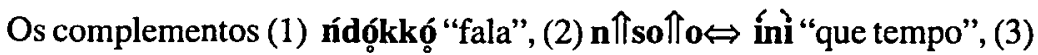
ńdídiá "(uma) comida", (4) $\mathbf{u} \exists \mathbf{r u} \exists \mathbf{a} \exists$ "mercado" são diferentes do complemento (5) mîn "me". O complemento (5) míen "me" checa seus traços- $\varphi$ de concordância do objeto em TP e ainda permanece visível em PF como parte da morfologia verbal. Os complementos verbais em (1)-(4) não têm seus traços- $\varphi$ de concordância do objeto visíveis em PF.

Logo, uma pergunta que deve ser feita é: por que os OAfs do complemento (5) mien "me" permanecem visíveis em PF, mas os OAfs dos outros complementos verbais em (1)-(4) não permanecem visíveis em PF?

Uma possível resposta para a questão colocada acima pode ser obtida através da análise dos tipos semânticos dos complementos DPs. Como verbos transitivos são funções, apresentam-se, em (1a)-(5a), os tipos semânticos dos complementos DPs (1)-(5):

a. (Enọ) á-dọkkoó ńdọkkơ

Eno fala a fala

a'. $\langle\mathrm{e}\langle\mathrm{e}, \mathrm{b}\rangle\rangle$

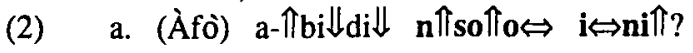

Você virá quando/que tempo?

a'. $\langle e<e, t\rangle$

8 Com exceção das orações imperativas. 
(3) a. (Èmè) á-nâm rídídíá Eme está cozinhando (uma) refeição a'. $\langle e<e, \downarrow\rangle>$

(4) a. (Àfò) à-ka\& $\mathbf{u} \exists r u \exists a \exists$ Você vai ao mercado $\mathrm{a}^{\prime} .\langle\mathrm{e}\langle\mathrm{e}, \mathrm{b}\rangle\rangle$
a. (Òkón) à-ké ì̀-kooód (mín) (Okon) chamou (me)
$a^{\prime} .\left\langle\mathbf{e}_{[+ \text {Humano }]}\langle\mathrm{e}, \mathrm{t}\rangle\right\rangle$

Os tipos semânticos ilustrados acima em (1 $\left.\mathrm{a}^{\prime}\right)$-(5a') demonstram que o complemento DP (5a') míen "me" é mais complexo que os DPs complementos (1a')-(4a'). Propõe-se que os traços de complemento do verbo em (5a) são checados no núcleo de mien "me", por combinarem exatamente com o traço de complemento [+HUMANO] de míen "me". A hipótese é elaborada abaixo em (6a)-(6b):
a. (Òkón) à-ké î̀-kooód (mîen)
(Okon) 3sSAf-pstll IsOAf-call (1s)
"(Okon) chamou (me)"

(6)b.

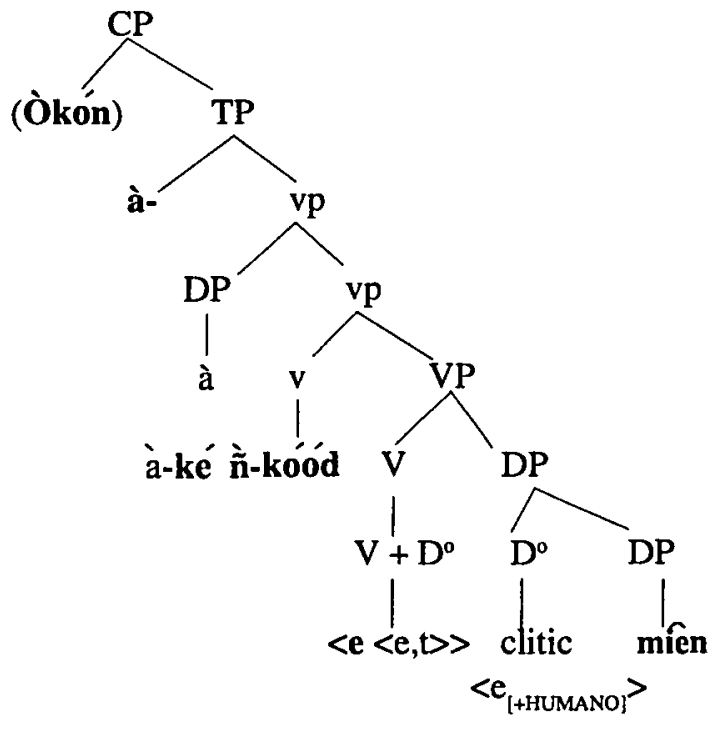


Advoga-se que o complemento DP (6a) mîn "me" é um DP complexo. O núcleo desse DP complexo é um clítico com o traço $\left\langle\mathrm{e}_{[+ \text {HUMANo }]}>\right.$. Esse clítico com o traço $<e_{[+ \text {HUMANo }]}>$ é incorporado no verbo e torna-se visível na morfologia do VP. Outro aspecto da proposta é de que o núcleo do especificador de VP seja o clítico sujeito. Neste caso, se um DP explícito encontra-se presente na oração, como Okon em (6a), esse DP ocupará então uma posição mais alta na oração, possivelmente uma posição na projeção $\mathrm{CP}$.

\section{Conclusão}

A análise acima, enfocada em modelos teóricos gerativos (semântico e sintático), propõe que o traço semântico [+HUMANO], quando presente na função VP em algum complemento DP em ibíbio, aciona um OAf, tornando-o visível em PF. A propriedade [+HUMANO] destes complementos DPs tem, portanto, um papel importante na interpretação semântica da língua ibíbio.

\section{RESUMO}

O objetivo deste estudo é mostrar que os traços-phi de concordância do sujeito SAfs -, quando checados na projeção de Tempo, sempre permanecem visíveis em Forma Fonológica $(\mathrm{PF})$ como parte da morfologia verbal na língua ibíbio. Por outro lado, os traços-phi de concordância do objeto - OAfs - só permanecem visíveis em PF quando o complemento Sintagma Determinante (DP) possui o traço semântico [+HUMANO].

Palavras-chave: ibibio, traços-phi de concordância do objeto, traço semântico [+HUMANO].

\section{ABSTRACT}

The purpose of this study is to show that the subject AGR phi-features SAfs when checked on TP always remain visible at PF as part of the verbal morphology in the Ibibio language. However, the object AGR phi-features OAfs only remain visible at $P F$ when the DP complement has the semantic feature [+HUMAN].

Key-words: ibibio, object agreement phi-features, semantic feature [+HUMAN]. 


\section{REFERÊNCIAS}

CLEMENTS, G. N. Phonology. In: HEINE, B.; NURSE, D. (Ed.). African languages: an introduction. Cambridge: Cambridge University Press, 2000. p. 123-160.

CHOMSKY, N. A.. Minimalist program. Cambridge, Mass.: MIT Press, 1995.

. Minimalist inquiries: the framework. Cambridge, Mass.: MIT, 1998. Occasional Papers in Linguistics, 15.

Derivation by phase. Cambridge, Mass.: MIT, 1999. Occasional Papers in Linguistics, 18.

CREISSELS, D. Typology. In: HEINE, B.; NURSE, D. (Ed.). African languages: an introduction. Cambridge: Cambridge University Press, 2000. p. 231- 258.

DICKSON, A. A. The case of the bound object complement in Ibibio. In: ANNUAL CONFERENCE OF THE LINGUISTIC ASSOCIATION OF NIGERIA (LAN), 14., 1995, Ado-Ekiti. Papers... Ado-Ekiti: Ondo State University, 1995.

ESSIEN, O. E. The ortography of Ibibio language. Nigeria: Ibibio Language Panel, 1983. . A grammar of the Ibibio language. Ibadan, Nigeria: University Press, 1990.

HEIM, I.; KRATZER, A. Semantics in generative grammar. Oxford, U.K.: Blakwell, 1998.

NDIMELE, O.; OLIVEIRA, M. S. D. de. An account of the missing subject and object in Ibibio. In: ANNUAL CONFERENCE ON AFRICAN LINGUISTICS - ACAL, 32., 2001, Berkeley-CA.

OLIVEIRA, M. S. D. de. A expressão do atributo na língua ibibio. Brasília, 1995. Dissertação (Mestrado) - Universidade de Brasília.

URUA, E. E. Object movement in Eastern Lower-Cross. In: DÉCHAINE, R.; MANFREDI, V. (Org.). Object positions in Benue-Kwa - Papers from a Workshop at Leiden University. The Netherlands: Holland Academic Graphics, 1997. p. 189-206.

. Ibibio phonetics and phonology. Cape Town: Centre for Advanced Studies of African Society, 2000.

WATTERS, J. R. Syntax. In: HEINE, B., NURSE, D. (Ed.) African languages. An introduction. Cambridge: Cambridge University Press, 2000. p. 194- 230. 
WILLIAMSON, K.; BLENCH, R. Niger-Congo. In: HEINE, B.; NURSE, D. (Ed.). African languages. An introduction. Cambridge: Cambridge University Press, 2000. p. 11-42.

\section{ANEXO 1 - MAPA-NIGÉRIA}

\section{NIGERIA ADMINISTRATIVE MAP}

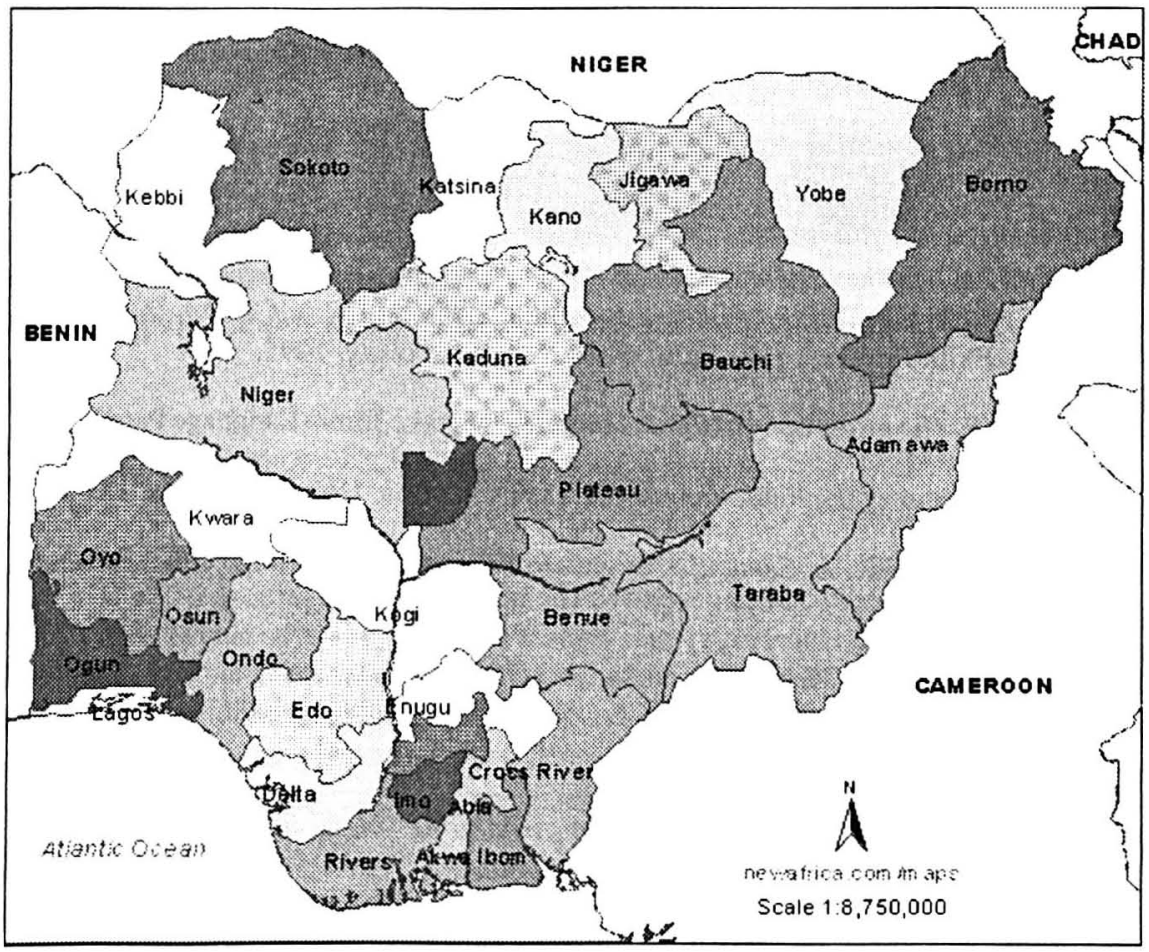

